\title{
ASSESSMENT OF OXIDATIVE STRESS AND ANTIOXIDANT STATUS AMONG PETROL STATIONS' WORKERS EXPOSED TO BENZENE IN ZAGAZIG CITY
}

\author{
Aida A. Hassan, Sahar A. Abou El-Magd, Amal F. Ghareeb* and Sarah A. Bolbol \\ Departments of Public Health and Biochemistry*, Faculty of Medicine, Zagazig University
}

\begin{abstract}
Background: The main environmental source of benzene exposure is vehicle exhaust emissions and evaporation losses of petrol at petrol filling stations. Activation of benzene and its reactive metabolites leads to continuous production of reactive oxygen species (ROS), which damages DNA, RNA, and proteins by chemical reactions. Aim \& Objectives: This study aims to promote health of petrol stations' workers occupationally exposed to benzene in their daily work with the following objectives: (1)Assess trans,trans-muconic acid (ttMA) level in urine as a biological marker for benzene exposure (2) Estimate the extent of oxidative stress by measuring the Malondialdehyde (MDA) level in blood (3) Assess the activity of erythrocytes superoxide dismutase (SOD) enzyme (4) Identify the personal and occupational risk factors that may be associated with level of (MDA) detected, activity of (SOD) enzyme and level of (ttMA) in studied groups. Subjects \& Methods: A cross sectional study was conducted among 43 male workers exposed to Benzene in 6 petrol stations present in Zagazig City. A control group of 40 male service workers, not exposed to Benzene were selected from the Faculty of Medicine in Zagazig University. All workers were subjected to a structured questionnaire included questions about (Personal and socio-demographic data- Occupational history- Oxidative stress questionnaire) and laboratory investigations to measure Trans,trans-muconic acid, Malondialdehyde and Superoxide Dismutase. Results: Results of this study showed that there is a high statistically significant difference regarding the level of (ttMA) \& (MDA) which is higher among exposed compared to control group, while the level of (SOD) is statistically lower among exposed group. There is a high statistical significant difference concerning the level of (MDA) among smokers and non-smokers in exposed group as it is higher among smokers compared to non-smokers. Results also showed that there is statistically significant difference regarding the effect of work duration on the level of (ttMA), (MDA) and (SOD) among exposed group, as levels of (ttMA) \& (MDA) increased with increased work duration while (SOD) level decreased with increased work duration. There is also a high statistically significant difference between level of (MDA) and chronic emotional stress among exposed group as (MDA) level increased with the increase of emotional stress. Conclusion: It was clear from this study that refueling procedure is an important source of exposure to benzene for the filling station workers who had higher level of benzene metabolites than control and the study also supports the hypothesis that Benzene and its metabolites induce oxidative stress which may plays a role in benzeneinitiated toxicity.
\end{abstract}

Keywords: Oxidative stress, Antioxidants, Petrol station, Benzene

\section{INTRODUCTION}

$\mathbf{P}$ etrol chemical is a complex combination of hydrocarbons, about $95 \%$ of compounds in petrol vapors are aliphatic and alicylic compounds and less than $2 \%$ are aromatics. The volatile nature of petrol makes it readily available in the atmosphere at any time it is dispensed, especially at petrol filling stations ${ }^{(1)}$.

Benzene (C6H6) is a volatile organic compound (VOC) with a very stable ring-shaped chemical structure that constitutes the base of the aromatic hydrocarbon family. It is a natural component of crude oil (less than $1 \%$ by weight) and is thus found in certain refined products, such as petrol (or gasoline) ${ }^{(2)}$. A main environmental source of benzene exposure is vehicle exhaust emissions and evaporation losses during the handling, distribution and storage of petrol ${ }^{(3)}$.

Oxidative stress is defined as an impaired balance between free radical production and antioxidant capacity resulting in excess oxidative products ${ }^{3}$. Malondialdehyde (MDA), which is an end product of the oxidation of polyunsaturated fatty acids has been used as a marker for oxidative stress as it can determine the degree of lipid peroxidation ${ }^{(4)}$.
Under normal conditions, scavenging molecules known as antioxidants convert ROS to $\mathrm{H} 2 \mathrm{O}$ to prevent overproduction of ROS. There are two types of antioxidants in the human body: enzymatic and non-enzymatic antioxidants. Enzymatic antioxidants are composed of superoxide dismutase, catalase, glutathione peroxidase and glutathione reductase, which also causes reduction of hydrogen peroxide to water and alcohol. Non-enzymatic antioxidants are also known as synthetic antioxidants or dietary supplements ${ }^{(5)}$.

Activation of benzene and its reactive metabolites leads to continuous production of reactive oxygen species (ROS), which damages DNA, RNA, and proteins by chemical reactions such as oxidation, nitration, and halogenations leading to genetic modification and alterations in the functions of important enzymes and proteins ${ }^{(6)}$.

Thus, this study aims to promote health of petrol stations' workers occupationally exposed to benzene in their daily work with objectives to (1) Assess trans,trans-muconic acid (ttMA) level in urine as a biological marker for benzene exposure (2) Estimate the extent of oxidative stress by measuring the Malondialdehyde (MDA) level in 
blood (3) Assess the activity of erythrocytes superoxide dismutase (SOD) enzyme (4) Identify the personal and occupational factors that may be associated with level of Malondialdehyde (MDA) detected, activity of superoxide dismutase (SOD) enzyme and level of trans,trans-muconic acid (ttMA) in studied groups.

\section{SUBJECTS AND METHODS}

\section{Technical design:}

Study design: A cross sectional study was conducted among petrol station workers in Zagazig city in Sharkia Governorate.

\section{Target groups:}

(1) Exposed group: It includes 40 workers at Petrol stations in Zagazig City exposed to Benzene on performing their job and,

(2) Non-exposed group: It includes 40 service workers not exposed to Benzene at their current occupation nor even had a past occupational history of exposure to Benzene. They were selected from the Faculty of Medicine at Zagazig University.

\section{Study population:}

Exposed group were 43 workers all were included in the study, they represents all Petrol station workers in 6 stations present in Zagazig City, 3 workers refused to participate in the study so only 40 workers agreed to participate.

Another group of 40 service workers, were selected from the Faculty of Medicine in Zagazig University and considered as a control group and both groups were male workers. Both exposed and control group were comparable as regards sex (all were males), age, residence.

Inclusion criteria: All exposed were male workers in Zagazig city, none of the workers included in this study had known exposure in any industry directly involved with benzene other than their current work. The subjects were apparently healthy between the age group of 18-58 years, with a minimum exposure of six months. The controls on the other hand were also males employed away from the petrol pumps and healthy. They were between 18 and 58 years with no exposure to petroleum fumes and matched for lifestyle (e.g., smoking) with exposed workers.

\section{Operational design:}

A. Data collection: A structured questionnaire was used to collect information from all participants about the following:

- Part one: included questions about Personal and socio-demographic data: age, residence, level of education, marital status and smoking habits. III.

- Part two: included questions about Occupational history (Current and previous occupation, Duration of employment in current occupation, Type of chemicals the worker exposed to, No. of hours worked/day, Presence of a side job or hobbies, Use of personal protective equipment)

- Part three: Oxidative stress questionnaire adapted from Holly questionnaire with little modification ${ }^{(7)}$.

\section{B. Laboratory investigations:}

(1) Assay of Trans,trans-muconic acid (ttMA): Assay of t,t-MA concentrations in the urine sample was done according to the methods of Boogaard and Van Sitter ${ }^{(8)}$. To improve the recovery, urinary samples were brought to $\mathrm{pH} 10$ by the addition of $35 \%$ sodium hydroxide aqueous solution. The reference range of trans, transmuconic acid in non-exposed subjects was $0.02-$ $0.338 \mathrm{mg} / \mathrm{L}^{(9)}$.

(2) Assay of Oxidative stress (Lipid Peroxide Malondialdehyde): Venous blood samples collected in plain glass tubes without using an anticoagulant and was left to clot for $30 \mathrm{~min}$. at $25^{\circ}$ C, the blood was centrifuged at 4,000 rpm for 15 min. at $4^{\circ} \mathrm{C}$. The top yellow serum layer was pipette off without distributing the white buffy layer. Serum level of MDA was measured according to the method of Ohkawa et al. ${ }^{(\mathbf{1 0})}$. The reference range of MDA was $0.12-1.71 \mathrm{nmol} / \mathrm{ml}$ (11).

(3) Assay of Antioxidants activity (Superoxide Dismutase SOD): Venous blood samples collected in K-EDTA tubes is used. Erythrocytes washed four times with $0.9 \% \mathrm{NaCl}$ solution. $0.5 \mathrm{ml}$ of whole blood centrifuged for 10 minutes at 4000 rpm and then the plasma was aspirate off. Erythrocytes then washed four times with $3 \mathrm{ml}$ of $0.9 \% \mathrm{NaCl}$ solution centrifuging for 10 minutes at $4000 \mathrm{rpm}$ after each wash. The washed centrifuged erythrocytes then made up to $2.0 \mathrm{ml}$ with cold redistilled water, mixed and left to stand at $+4^{\circ} \mathrm{C}$ for 15 minutes. The lysate is diluted with distilled water so that the \% inhibition falls between $30 \%$ and $60 \%$. The activity of SOD was assayed by spectrophotometry following the method originally developed by Nishikimi ${ }^{(12)}$ and then modified by Kakkar ${ }^{(13)}$. The reference range of SOD was 164 $-240 \mathrm{u} / \mathrm{ml}^{(\mathbf{1 4})}$.

C. Pilot study:

A pilot study was conducted during December 2011 to develop and check the questionnaire with the most appropriate and understandable terms and test the response to different items of the questionnaire. The pilot sample was 10 workers and they were not included in the study.

\section{Administrative design and Ethical issues:}

The ethical committee of the Zagazig faculty of Medicine approved the study protocol. Ethical considerations and confidentiality were respected. 
An informed consent was obtained from all participants of this study. The workers were told about the aim of the study, and they were informed that the data would be used for scientific purposes only. The workers were also given the right to refuse or participate in the study.

\section{Data management:}

The collected data were computerized and statistically analyzed using SPSS program (Statistical Package for Social Science) version 16.0 ${ }^{(\mathbf{1 5})}$. For the statistical calculations Data coding was done, and Qualitative data were represented as frequencies and percentages, Chi-square test $\left(\square^{2}\right)$, chi-square for trend and Fisher Exact test was carried out for testing the association between the qualitative data whenever possible. Quantitative data were compared using student's t-test and Mann-Whitney test. The test results were considered significant when p-value $<\mathbf{0 . 0 5}$.

\section{RESULTS}

Results of this study showed that the exposed and control groups are comparable regarding age, marital status, the level of education and residence and living conditions (Table 1). Workers are also comparable regarding smoking habits. More than half of the exposed group worked for less than 10 years $(62.5 \%)$, while more than half of the control group worked for more than 10 years $(52.5 \%)$.

\section{Oxidative stress questionnaire results in studied} groups

Results of this study showed no statistical significant difference ( $\mathrm{p}$-value $>0.05$ ) as regards to the life style habits, eating of fruits and vegetables, taking nutritional supplements and living conditions in both exposed and control groups. Regarding the level of work stress results of this study showed no statistical significant difference (p-value > 0.05) among both exposed and control groups, while the exposed group experienced higher exposure to harmful chemical substances than control group ( $\mathrm{p}$-value < 0.01) (Table 2)

Results of this study showed no statistical significant difference (p-value > 0.05) regarding suffering from chronic emotional stress, allergic tendencies, infections and aging symptoms, also using of several rounds of antibiotics among exposed and control groups. There is also no statistical significant difference ( $p$-value $>0.05$ ) as regards to having metal felling, use of fluorinated toothpaste/mouth wash and frequency of radiological investigations among exposed and control groups.

Benzene exposure metabolite and Oxidative stress biomarkers

Results of this study showed that there is high statistically significant difference $(p$-value $<0.01)$ regarding the level of trans ,trans-muconic acid (ttMA) \& malondialdehyde (MDA) among exposed and control groups as it is higher among exposed group, while the level of superoxide dismutase (SOD) is lower among exposed group (Table3). Study results also showed significant relation between the levels of Malondialdehyde (MDA) (strong positive relation), superoxide dismutase (SOD) (negative relation) and Level of trans ,Trans-muconic acid (ttMA) (Figure 2, 3).

This study also showed that on studying the association of age on the level of trans,transmuconic acid (ttMA), malondialdehyde (MDA) \& superoxide dismutase (SOD) in exposed group there was no statistically significant difference (pvalue > 0.05) among the two age groups (Table 4).

Results of this study showed that there is a higher statistical significant difference (p-value < $0.01)$ concerning the level of malondialdehyde (MDA) being higher among exposed smokers compared to exposed non-smokers, also there is lower statistical significant difference ( $\mathrm{p}$-value $<0.05)$ regarding the level of superoxide dismutase (SOD) as it was lower in exposed smokers than exposed non-smokers (Table 5).

This study showed that there is no statistical significant difference ( $\mathrm{p}$-value $>0.05$ ) as regards to the relation between the number of smoked cigarettes, smoking duration in exposed group and the levels of trans,trans-muconic acid (ttMA) \& malondialdehyde (MDA) \& superoxide dismutase (SOD).

There is high statistical difference ( $p$-value < 0.01 ) regarding the effect of work duration on the level of trans,trans-muconic acid (ttMA), malondialdehyde (MDA) and superoxide dismutase (SOD) as both ttMA and MDA levels increased with increase work duration, while SOD level decreased with increased work duration (Table 6).

\section{Association between oxidative stress questionnaire and level of oxidative stress markers in exposed group}

There is no statistically significant difference concerning the sleeping habits, eating of fruits and vegetables, taking nutritional supplements and its relation to the level of malondialdehyde (MDA) \& the activity of superoxide dismutase (SOD). Results also showed that there is no statistically significant difference ( $p$-value $>0.05$ ) concerning the level of malondialdehyde (MDA) \& the level of superoxide dismutase (SOD) and their relation to living conditions and work environment.

Results of the study showed that there is high statistically significant difference ( $p$-value $<0.01$ ) concerning the level of malondialdehyde (MDA) 
and its relation to chronic emotional stress as level of MDA increased with increased emotional stress while there is no statistical significant difference
( $\mathrm{p}$-value $>0.05$ ) concerning the level of superoxide dismutase (SOD) and its relation to petrol station workers health conditions (Table 7, 8).

Table (1): Frequency distribution of some socio-demographic characteristics and living conditions of petrol station workers and control group

\begin{tabular}{|c|c|c|c|c|c|c|}
\hline \multirow{2}{*}{$\begin{array}{c}\text { Socio demographic } \\
\text { characteristics \& Living } \\
\text { conditions }\end{array}$} & \multicolumn{2}{|c|}{$\begin{array}{c}\text { Exposed group } \\
\qquad=40\end{array}$} & \multicolumn{2}{|c|}{$\begin{array}{c}\text { Control group } \\
\mathbf{N}=\mathbf{4 0}\end{array}$} & \multirow[t]{2}{*}{$\chi^{2}$} & \multirow[t]{2}{*}{$P$ value } \\
\hline & $\mathbf{n}$ & $\%$ & $\mathbf{n}$ & $\%$ & & \\
\hline \multicolumn{7}{|l|}{ age } \\
\hline $18 y-<40 y$ & 30 & 75 & 25 & 62.5 & 1.45 & 0.228 \\
\hline $40 y-60 y$ & 10 & 25 & 15 & 37.5 & & \\
\hline \multicolumn{7}{|l|}{-Marital status: } \\
\hline Married & 33 & 82.5 & 36 & 90 & 0.949 & 0.330 \\
\hline Not married & 7 & 17.5 & 4 & 10 & & \\
\hline \multicolumn{7}{|l|}{-Level of education: } \\
\hline Illiterate & 4 & 10 & 6 & 15 & & \\
\hline read and write & 9 & 22.5 & 13 & 32.5 & $2.148 *$ & 0.14 \\
\hline school education & 18 & 45 & 16 & 40 & & \\
\hline Higher education & 9 & 22.5 & 5 & 12.5 & & \\
\hline \multicolumn{7}{|l|}{-Residence } \\
\hline Urban & 5 & 12.5 & 7 & 17.5 & 0.392 & 0.531 \\
\hline Rural & 35 & 87.5 & 33 & 82.5 & & \\
\hline \multicolumn{7}{|l|}{ Living in high polluted area } \\
\hline \multirow[t]{2}{*}{ No } & 25 & 62.5 & 22 & 55 & 0.464 & 0.496 \\
\hline & 15 & 37.5 & 18 & 45 & & \\
\hline \multicolumn{6}{|l|}{ EUsing sprays for pest } & 0.822 \\
\hline Yes & 23 & 42.5 & 18 & 55 & 0.051 & \\
\hline No & 17 & & & 45 & & \\
\hline \multicolumn{7}{|l|}{ Esing household cleaning } \\
\hline Yes & 28 & 30 & 17 & 57.5 & 1.352 & \\
\hline No & 12 & & & 42.5 & & \\
\hline
\end{tabular}

$* \chi 2$ for linear trend

Table (2): Work environment in both exposed and non-exposed participants

\begin{tabular}{|c|c|c|c|c|c|c|}
\hline \multirow{2}{*}{ Work environment } & \multicolumn{2}{|c|}{$\begin{array}{c}\text { Exposed group } \\
N=40\end{array}$} & \multicolumn{2}{|c|}{$\begin{array}{c}\text { Control group } \\
N=40\end{array}$} & \multirow[t]{2}{*}{$\chi^{2}$} & \multirow{2}{*}{$P$ value } \\
\hline & $\mathbf{n}$ & $\%$ & $\mathbf{n}$ & $\%$ & & \\
\hline $\begin{array}{l}\text {-Level of stress in your job } \\
\text { Mild } \\
\text { Moderate }\end{array}$ & $\begin{array}{c}9 \\
31\end{array}$ & $\begin{array}{l}22.5 \\
77.5\end{array}$ & $\begin{array}{c}8 \\
32\end{array}$ & $\begin{array}{l}20 \\
80\end{array}$ & 0.07 & 0.78 \\
\hline $\begin{array}{l}\text { - Level of your exposure to } \\
\text { harmful chemical substances } \\
\text { Moderate } \\
\text { Severe }\end{array}$ & $\begin{array}{l}10 \\
30\end{array}$ & $\begin{array}{l}25 \\
75\end{array}$ & $\begin{array}{c}40 \\
0\end{array}$ & $\begin{array}{c}100 \\
0\end{array}$ & $\begin{array}{l}\text { Fisher } \\
\text { exact }\end{array}$ & $0.000 *$ \\
\hline
\end{tabular}

* High statistical difference $p$-value $<0.01$ 
Table (3): Association between level of trans ,trans-muconic acid (ttMA) \& malondialdehyde (MDA) \& superoxide dismutase (SOD) in both exposed and non exposed participants

\begin{tabular}{lcccc}
\hline \multicolumn{1}{c}{ Biomarkers } & $\begin{array}{c}\text { Exposed group } \\
\mathbf{N = 4 0}\end{array}$ & $\begin{array}{c}\text { Control group } \\
\mathbf{N = 4 0}\end{array}$ & $\begin{array}{c}\text { Mann- } \\
\text { Whitney } \\
\text { test }\end{array}$ & P value \\
\cline { 2 - 5 } & Median (range) & Median (range) & 199.000 & $0.000^{*}$ \\
\hline $\begin{array}{l}\text { Trans ,trans-muconic } \\
\text { acid (ttMA) }\end{array}$ & $0.50(0.21-1.80)$ & $0.18(0.01-0.57)$ & & \\
Malondialdehyde (MDA) & $9.74(1.09-61.28)$ & $1.55(0.26-11.95)$ & 296.500 & $0.000^{*}$ \\
$\begin{array}{l}\text { Superoxide dismutase } \\
\text { (SOD) }\end{array}$ & $2.66(120.10-338.60)$ & $3.02(283.40-356.80)$ & 196.500 & $0.000^{*}$ \\
\hline
\end{tabular}

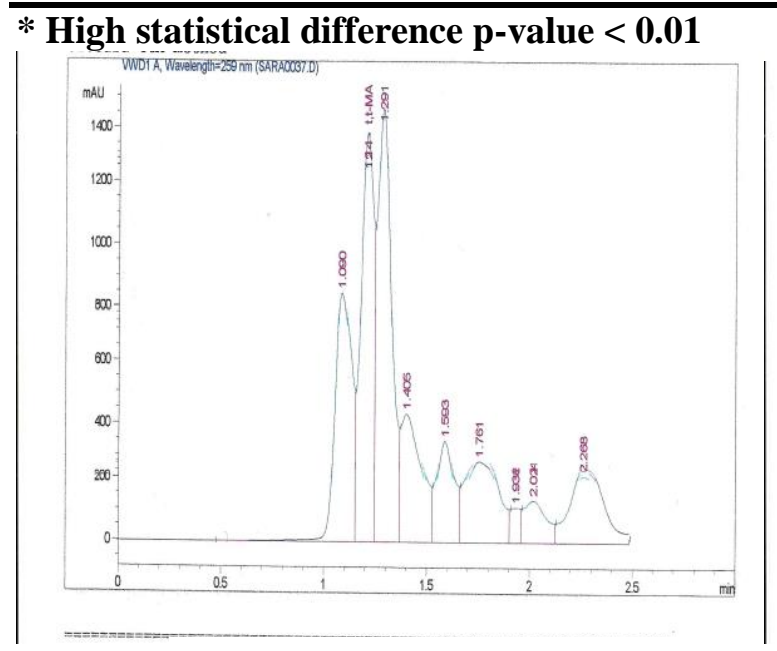

Exposed worker

Level of urinary ttMA $1.8 \mathrm{mg} / \mathrm{L}$

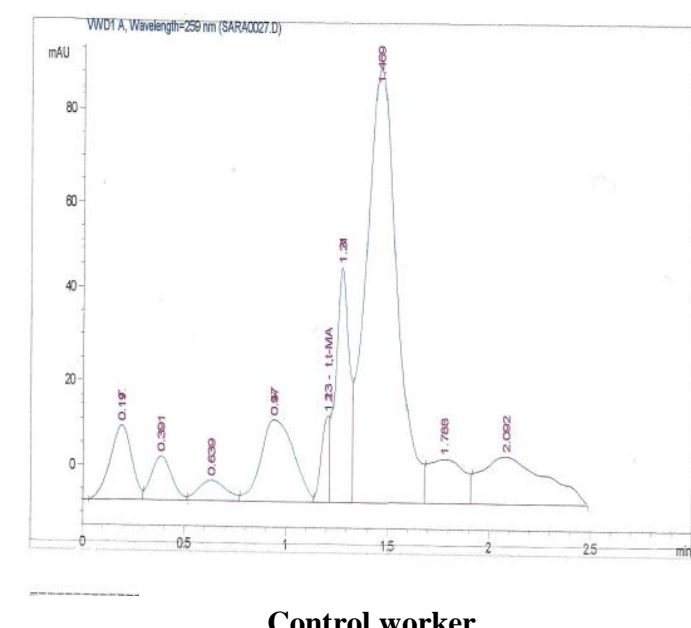

Level of urinary ttMA 0.01 $\mathbf{m g} / \mathbf{L}$

Figure (1): Urinary excretion of trans ,trans-muconic acid (ttMA) in Exposed \& control workers as shown by HPLC

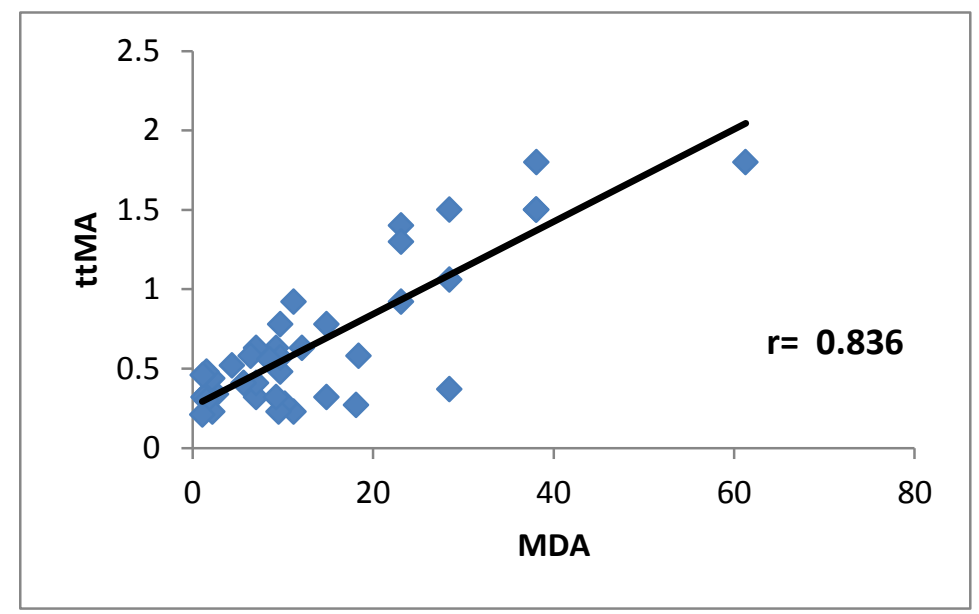

Figure (2):

This line graph shows significant relation (positive strong relation) between the level of malondialdehyde (MDA) and Level of trans, transmuconic acid (ttMA)

P value 0.000

Figure (2): Association between malondialdehyde (MDA) and Level of trans ,trans-muconic acid (ttMA) in exposed group. 


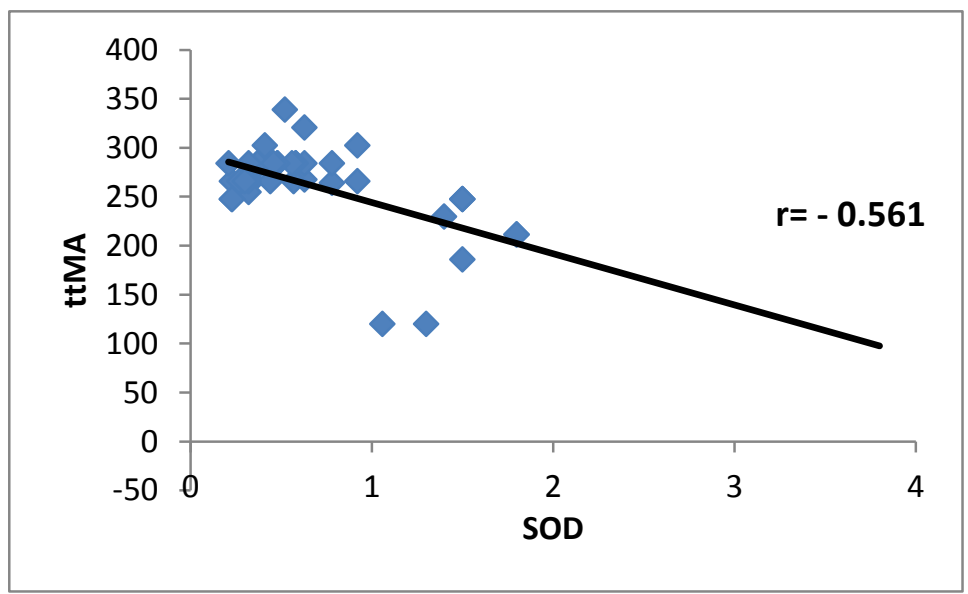

Figure (3):

This line graph shows significant relation (negative relation) between the level of superoxide dismutase (SOD) and Level of trans,trans-muconic acid (ttMA)

P value 0.000

Figure (3): Association between superoxide dismutase (SOD) and Level of trans ,trans-muconic acid (ttMA) in exposed group.

Table (4): Association between age in petrol station workers and Level of trans,trans-muconic acid (ttMA) \& malondialdehyde (MDA) \& superoxide dismutase (SOD)

\begin{tabular}{|c|c|c|c|c|}
\hline \multirow{3}{*}{ Biomarkers } & \multicolumn{2}{|c|}{$\begin{array}{c}\text { Age } \\
\text { Exposed group }(\mathrm{N}=40) \\
\end{array}$} & \multirow{3}{*}{$\begin{array}{c}\text { Mann- } \\
\text { Whitney } \\
\text { test }\end{array}$} & \multirow[t]{3}{*}{$P$ value } \\
\hline & $20-<40(n=30)$ & $40-60(n=10)$ & & \\
\hline & Median (Range) & Median (Range) & & \\
\hline $\begin{array}{l}\text { Trans ,trans-muconic acid } \\
\text { (ttMA) }\end{array}$ & $0.45(0.23-1.80)$ & $0.71(0.21-1.50)$ & 573.500 & 0.236 \\
\hline Malondialdehyde (MDA) & $9.27(1.16-61.28)$ & $16.42(1.09-38.09)$ & 135.500 & 0.650 \\
\hline \multirow[t]{2}{*}{ Superoxide dismutase (SOD } & & & & \\
\hline & $2.66(120.10-338.60)$ & $2.66(120.10-320.40)$ & 135.000 & 0.632 \\
\hline
\end{tabular}

Table (5): Association between smoking habits in petrol station workers and Level of trans,trans-muconic acid (ttMA) \& malondialdehyde (MDA) \& superoxide dismutase (SOD)

\begin{tabular}{|c|c|c|c|c|}
\hline \multirow{3}{*}{ Biomarkers } & \multicolumn{2}{|c|}{$\begin{array}{c}\text { Smoking habits } \\
\text { Exposed group }(\mathrm{N}=40) \\
\end{array}$} & \multirow{3}{*}{$\begin{array}{c}\text { Mann- } \\
\text { Whitney } \\
\text { test }\end{array}$} & \multirow[t]{3}{*}{$P$ value } \\
\hline & Smoker $(n=22)$ & Non-smoker $(\mathrm{n}=18)$ & & \\
\hline & Median (range) & Median (range) & & \\
\hline $\begin{array}{l}\text { Trans ,trans-muconic acid } \\
\text { (ttMA) }\end{array}$ & $0.58(0.21-1.80)$ & $0.48(0.23-1.40)$ & 193.500 & 0.904 \\
\hline Malondialdehyde (MDA) & $14.84(1.09-61.28)$ & $6.76(1.16-23.09)$ & 89.500 & $0.003 * *$ \\
\hline \multirow[t]{2}{*}{ Superoxide dismutase (SOD) } & & & & $0.022^{*}$ \\
\hline & $2.66(120.10-320.40)$ & $2.84(120.10-338.60)$ & 115.500 & \\
\hline
\end{tabular}

\footnotetext{
*p-value $<0.05$

$* *$ High statistical difference p-value $<0.01$
} 
Table (6): Association between petrol station workers work duration and level of trans ,trans-muconic acid (ttMA) \& malondialdehyde (MDA) \& superoxide dismutase (SOD)

\begin{tabular}{|c|c|c|c|c|}
\hline \multirow{3}{*}{ Biomarkers } & \multicolumn{2}{|c|}{$\begin{array}{l}\text { Work duration in Exposed group } \\
\qquad(\mathrm{N}=40)\end{array}$} & \multirow{3}{*}{$\begin{array}{c}\text { Mann- } \\
\text { Whitney } \\
\text { test }\end{array}$} & \multirow[t]{3}{*}{$P$ value } \\
\hline & $\begin{array}{c}<10 \text { years } \\
(\mathrm{n}=25)\end{array}$ & $\begin{array}{c}\geq 10 \text { years } \\
(\mathrm{n}=15)\end{array}$ & & \\
\hline & Median (range) & Median (range) & & \\
\hline $\begin{array}{l}\text { Trans ,trans-muconic acid } \\
\text { (ttMA) }\end{array}$ & $0.41(0.23-0.92)$ & $1.06(0.21-1.80)$ & 92.000 & $0.008 * *$ \\
\hline Malondialdehyde (MDA) & $9.27(1.16-28.45)$ & $23.09(1.09-61.28)$ & 110.000 & $0.030 *$ \\
\hline Superoxide dismutase (SOD) & $2.84(247.60-338.60)$ & $2.48(120.10-283.90)$ & 74.000 & $0.001 * *$ \\
\hline
\end{tabular}

\footnotetext{
*p-value $<0.05$

** High statistical difference p-value $<0.01$
}

Table (7): Association between petrol station workers health conditions and level of malondialdehyde (MDA)

\begin{tabular}{|c|c|c|c|c|c|c|c|}
\hline \multirow{3}{*}{ Health conditions } & \multicolumn{4}{|c|}{ Malondialdehyde (MDA) } & \multirow{3}{*}{$\begin{array}{c}\text { Fisher } \\
\text { exact }\end{array}$} & \multirow{3}{*}{$\begin{array}{c}\mathbf{P} \\
\text { value }\end{array}$} & \multirow{3}{*}{$\begin{array}{c}\text { OR } \\
(95 \% \mathrm{CI})\end{array}$} \\
\hline & \multicolumn{2}{|c|}{$\geq 2.0 \mathrm{nmol} / \mathrm{L}$} & \multicolumn{2}{|c|}{$<2.0 \mathrm{nmol} / \mathrm{L}$} & & & \\
\hline & $\mathbf{N}$ & $\%$ & $\mathbf{N}$ & $\%$ & & & \\
\hline $\begin{array}{l}\text {-Having chronic } \\
\text { emotional stress } \\
\text { Yes } \\
\text { No }\end{array}$ & $\begin{array}{l}21 \\
13\end{array}$ & $\begin{array}{l}100 \\
68.4\end{array}$ & $\begin{array}{l}0 \\
6\end{array}$ & $\begin{array}{c}0 \\
31.6\end{array}$ & - & $0.007^{*}$ & $\begin{array}{c}0.684 \\
(0.504-0.929)\end{array}$ \\
\hline $\begin{array}{l}\text {-Having allergic } \\
\text { tendencies } \\
\text { Yes } \\
\text { No }\end{array}$ & $\begin{array}{l}11 \\
23\end{array}$ & $\begin{array}{l}91.7 \\
82.1\end{array}$ & $\begin{array}{l}1 \\
5\end{array}$ & $\begin{array}{c}8.3 \\
17.9\end{array}$ & - & 0.648 & $\begin{array}{c}0.418 \\
(0.043-4.024)\end{array}$ \\
\hline $\begin{array}{l}\text { Suffering from infections } \\
\text { Sometimes } \\
\text { Often } \\
\text { Very often }\end{array}$ & $\begin{array}{c}25 \\
6 \\
3\end{array}$ & $\begin{array}{l}87.1 \\
83.3 \\
66.7\end{array}$ & $\begin{array}{l}6 \\
0 \\
0\end{array}$ & $\begin{array}{l}12.9 \\
16.7 \\
33.3\end{array}$ & $1.721 * *$ & 0.189 & - \\
\hline $\begin{array}{l}\text {-Using several rounds of } \\
\text { antibiotics } \\
\text { Yes } \\
\text { No }\end{array}$ & $\begin{array}{l}17 \\
17\end{array}$ & $\begin{array}{l}94.4 \\
77.3\end{array}$ & $\begin{array}{l}1 \\
5\end{array}$ & $\begin{array}{c}5.6 \\
22.7\end{array}$ & - & 0.197 & $\begin{array}{c}0.200 \\
(0.021-1.897)\end{array}$ \\
\hline $\begin{array}{l}\text {-Suffering from aging } \\
\text { symptoms } \\
\text { Yes } \\
\text { No }\end{array}$ & $\begin{array}{c}8 \\
26\end{array}$ & $\begin{array}{l}100 \\
81.3\end{array}$ & $\begin{array}{l}0 \\
6\end{array}$ & $\begin{array}{c}0 \\
18.7\end{array}$ & - & 0.318 & $\begin{array}{c}0.812 \\
(0.688-0.960)\end{array}$ \\
\hline
\end{tabular}

* High statistical difference p-value $<0.01$

$* * \chi 2$ for linear trend 
Table (8): Association between petrol station workers health conditions and level of superoxide dismutase (SOD)

\begin{tabular}{|c|c|c|c|c|c|c|c|}
\hline \multirow{3}{*}{ Health conditions } & \multicolumn{4}{|c|}{ Superoxide dismutase (SOD) } & \multirow{3}{*}{$\begin{array}{c}\text { Fisher } \\
\text { exact }\end{array}$} & \multirow{3}{*}{$\begin{array}{c}\mathbf{P} \\
\text { value }\end{array}$} & \multirow{3}{*}{$\begin{array}{c}\text { OR } \\
(95 \% \mathrm{CI})\end{array}$} \\
\hline & \multicolumn{2}{|c|}{$<160 \mathrm{u} / \mathrm{ml}$} & \multicolumn{2}{|c|}{$\geq 160 \mathrm{u} / \mathrm{ml}$} & & & \\
\hline & $\mathbf{N}$ & $\%$ & $\mathbf{N}$ & $\%$ & & & \\
\hline $\begin{array}{l}\text { oHaving chronic } \\
\text { emotional stress } \\
\text { Yes } \\
\text { No }\end{array}$ & $\begin{array}{l}1 \\
1\end{array}$ & $\begin{array}{l}4.8 \\
5.3\end{array}$ & $\begin{array}{l}20 \\
18\end{array}$ & $\begin{array}{l}95.2 \\
94.7\end{array}$ & - & 0.219 & $\begin{array}{c}0.895 \\
(0.767-1.044)\end{array}$ \\
\hline $\begin{array}{l}\text { oHaving Allergic } \\
\text { tendencies } \\
\text { Yes } \\
\text { No }\end{array}$ & $\begin{array}{l}11 \\
27\end{array}$ & $\begin{array}{l}91.7 \\
96.4\end{array}$ & $\begin{array}{l}1 \\
1\end{array}$ & $\begin{array}{l}8.3 \\
3.6\end{array}$ & - & 0.515 & $\begin{array}{c}0.407 \\
(0.023-7.108)\end{array}$ \\
\hline $\begin{array}{l}\text {-Suffering from } \\
\text { infections } \\
\text { Sometimes } \\
\text { Often } \\
\text { Very often }\end{array}$ & $\begin{array}{l}1 \\
0 \\
1\end{array}$ & $\begin{array}{c}3.2 \\
0 \\
33.3\end{array}$ & $\begin{array}{c}30 \\
6 \\
2\end{array}$ & $\begin{array}{c}96.8 \\
100 \\
66.7\end{array}$ & $2.794 *$ & 0.09 & - \\
\hline $\begin{array}{l}\text { using several rounds } \\
\text { of antibiotics } \\
\text { Yes } \\
\text { No }\end{array}$ & $\begin{array}{l}1 \\
1\end{array}$ & $\begin{array}{l}5.6 \\
4.5\end{array}$ & $\begin{array}{l}17 \\
21\end{array}$ & $\begin{array}{l}94.4 \\
95.5\end{array}$ & - & 1.000 & $\begin{array}{c}0.810 \\
(0.047-13.920)\end{array}$ \\
\hline $\begin{array}{l}\text {-Suffering from aging } \\
\text { symptoms } \\
\text { Yes } \\
\text { No }\end{array}$ & $\begin{array}{l}0 \\
2\end{array}$ & $\begin{array}{c}0 \\
6.2\end{array}$ & $\begin{array}{c}8 \\
30\end{array}$ & $\begin{array}{l}100 \\
93.8\end{array}$ & - & 1.000 & $\begin{array}{c}0.938 \\
(0.857-1.025)\end{array}$ \\
\hline
\end{tabular}

\section{$* \chi 2$ for linear trend}

\section{DISCUSSION}

Trans,trans-Muconic acid (ttMA) which is a non-phenolic metabolite of benzene excreted into the urine, has been proposed as a specific indicator for monitoring benzene exposure ${ }^{(\mathbf{1 6})}$. Oxidative stress is defined as a disequilibrium between prooxidant and anti-oxidant systems, Plasma Malondialdehyde (MDA) is a biological marker of lipid peroxidation resulting from oxidative stress (17). Human body is equipped with various antioxidants like superoxide dismutase (SOD), glutathione peroxides (GSHPx) and catalase (CAT) which can counteract the deleterious action of ROS and protect from cellular and molecular damage ${ }^{(18)}$.

Results of this study showed that there is a high significant statistical difference regarding the level of trans ,Trans-muconic acid (ttMA) among petrol station workers compared to control group which is higher among exposed, and this is consistent with other studies that showed levels of benzene and its metabolites were significantly elevated among all gasoline filling workers compared to their control ${ }^{(6,19,20)}$.

This study showed high statistical significant difference among exposed group compared to control group regarding the level Malondialdehyde (MDA) which was higher among exposed. This is in agreement with other studies which illustrated that Benzene exposure has been associated with increases in the overall formation of MDA ${ }^{(21,22)}$, also this study is consistent with other studies which found a significant increase in the level of MDA in petrol station workers compared to their control group ${ }^{(6,19,23-25)}$. In addition, a study by Abdallah et al. ${ }^{(26)}$ also showed increased level of MDA among shoe makers exposed to petroleum products.

Results of this study showed that the level of superoxide dismutase (SOD) is lower among 
exposed group. This is consistent with other studies which showed that the activity of SOD were significantly lower in workers exposed to Benzene compared to control group ${ }^{(25)}$, also other studies ${ }^{(6,23,24)}$ found that SOD was significantly decreased in the Petrol filling workers compared to control group. Abdallah et al. ${ }^{(26)}$ also showed decreased level of SOD among shoe makers exposed to petroleum products.

Karagozler et al. ${ }^{(24)}$ also reported significantly lowered serum SOD activity in house painters exposed to organic solvents including benzene. Chen ${ }^{(21)}$ also reported that benzene exposure decreases the level of serum SOD in humans. Rekhadevi et al. ${ }^{(27)}$ also reported decreased level of SOD in filling station attendants exposed to petroleum Hydrocarbons. In contrast to our study Al-Helaly ${ }^{(28)}$ found non significant decrease in SOD level petrol station workers.

Results of this study showed significant relation between the levels of Malondialdehyde (MDA) (strong positive relation), superoxide dismutase (SOD) (negative relation) and the level of trans ,Trans-muconic acid (ttMA). These findings are in agreement with other studies ${ }^{(6,24)}$ that observed significant correlation between the concentration of benzene in the blood and elevated levels of MDA, also these studies showed that the level of SOD was significantly decreased in the study group exposed to benzene compared to controls. Kim et al. ${ }^{(29)}$ also found significant association between the level of MDA and biomarkers for Benzene, toluene, ethylbenzene, and xylene (BTEX).

On studying the effect of age on the level of Malondialdehyde (MDA) \& superoxide dismutase (SOD) there was no statistical significant difference among exposed and control groups and this is consistent with other studies that detected no significant age related increase in MDA ${ }^{(30)}$. But results is in contrast with another studies which showed that MDA concentrations increased with age ${ }^{(31)}$, and SOD level decreased with age ${ }^{32}$.

Tobacco smoke is a rich source of oxidants. It has been argued that, the increased production of reactive oxygen species associated with smoking may exceed the capacity of oxidant defense system, resulting in oxidative damage ${ }^{(33)}$. This study showed that there was a higher statistical significant difference among smokers concerning the level of Malondialdehyde (MDA) compared to non-smokers. These findings were in agreement with other studies which detected that Malondialdehyde levels were significantly increased in cigarette smokers ${ }^{(\mathbf{3 0 , 3 4 - 3 6})}$. Also there is lower statistical significant difference regarding the level of superoxide dismutase (SOD) among smokers compared to non-smokers. This was consistent with other studies that found decreased activity of SOD among smokers ${ }^{(34,35)}$.

Results of this study showed that there is a statistical significant difference regarding the effect of work duration on the levels of Trans,trans-muconic acid (ttMA), superoxide dismutase (SOD) and Malondialdehyde (MDA) among exposed group as the levels of ttMA \& MDA increased with increase work duration while the level of SOD decreased with increased work duration. This is in agreement with other studies that showed workers had significant uptake of benzene due to prolonged work hours and increased years of exposure which will also leads to the formation of ROS, decreases antioxidant activity and hence increases oxidative stress ${ }^{(6,17)}$.

Concerning the health conditions study results showed that most workers complaining of chronic emotional stress has significantly higher levels of Malondialdehyde (MDA) compared with those that don't complain of chronic emotional stress. This is in agreement with other studies which indicated that in stress condition oxidative damage to DNA and sensitivity to lipid oxidation were significantly increased when compared with the same parameters in "non-stress" conditions ${ }^{(37,38)}$. While in contrast with this study results which found no statistically significant difference as regards the relation between suffering of chronic emotional stress and the superoxide dismutase (SOD) activity level, Sivonová et al. ${ }^{(38)}$ found a significant decrease in plasma antioxidant activity in students who were under stress.

\section{CONCLUSION AND RECOMMENDATIONS}

It was clear from this study that refueling procedure is an important source of exposure to benzene for the filling station workers and this was obvious by the fact that petrol station workers had higher level of benzene metabolites than control persons working in the university who did not directly get in contact with benzene. Also this study supports the hypothesis that Benzene and its metabolites induce oxidative stress which was noticed by the results of the study that showed increased level of MDA and decreased antioxidants activity among the petrol station workers and this plays a role in benzene-initiated toxicity. So we recommend regular screening of workers by having annual blood tests to detect any abnormal blood counts, also Health education of workers on the negative health implication of exposure to the Benzene and consequences of smoking, Provide personal protective equipment 
(PPE) and Improving lifestyle and behavioral changes to avoid chronic stress.

\section{ACKNOWLEDGEMENTS}

We wish to thank all workers for their cooperation and help.

\section{REFERENCES}

1. Sellappa S, Sadhanandhan B, Francis A, et al. (2010): Evaluation of Genotoxicity in Petrol Station Workers in South India Using Micronucleus Assay. Industrial Health; 48:852856.

2. Schnatter R. (2000): Petroleum Worker Studies and Benzene Risk Assessment. journal of Toxicology and Environmental Health, Part A: Current Issues; 61(5-6): 433-437.

3. Hong Y, Park E, Park M, et al. (2009): Community level exposure to chemicals and oxidative stress in adult population. Toxicol Lett.;184(2):139-144.

4. Bae S, Pan X, Kim S, et al. (2010): Exposures to particulate matter and polycyclic aromatic hydrocarbons and oxidative stress in schoolchildren. Environ Health Perspect. ; 118(4):579-583.

5. Agarwal A, Gupta S and Sharma R. (2005): Role of oxidative stress in female reproduction. Reproductive Biology and Endocrinology; 14(3):28-53.

6. Uzma N, Kumar B and Hazari M. (2010): Exposure to Benzene Induces Oxidative Stress, Alters the Immune Response and Expression of p53 in Gasoline Filling Workers. American Journal of Industrial Medicine; 53:1264-1270.

7. Holly L. (2009): Healthy Aging. Retriverd from: http://www.ATDonline.org (Accessed date: December 28, 2009).

8. Boogaard P and van Sittert N. (1996): Suitability of S-Phenyl Mercapturic Acid and trans-transMuconic Acid as Biomarkers for Exposure to Low Concentrations of Benzene.Environ. Health. Perspect; 104(6):1151-1158.

9. Waidyanatha S, Rothman N, Li G, et al. (2004): Rapid determination of six urinary metabolites in occupationally exposed and unexposed subjects. Anal. Biochem. ;327(2):184-189.

10. Ohkawa H, Ohishi N and Yagi K. (1979): Assay for lipid peroxides in animal tissues by thiobarbituric acid reaction. Anal Biochem; 95(2):351-359.

11. Dixon Z, Shie F, Warden B, et al. (1998): The Effect of a Low Carotenoid Diet on Malondialdehyde-Thiobarbituric Acid (MDATBA) Concentrations in Women: A PlaceboControlled Double-Blind Study. Journal of the American College of Nutrition; 17(1):54-58.

12. Nishikimi M, Rao N and Yagi K. (1972): The occurrence of superoxide anion in the reaction of reduced phenazine methosulfate and molecular oxygen. Biochem.Biophys. Res. Commun. ; 46(2): 849-854.

13. Kakkar P, Das B and Viswanathan P. (1984): A modified spectrophotometric assay of superoxide dismutase. Indian J. Biochem. Biophys; 21(2):130132.
14. Fortress Diagnostics Limited Unit (United Kingdom). (2012): Superoxide Dismutase. Viewed Nov/2012. Revised AUG/2009. Available from: http://www.fortressdiagnostics.com/online/ifu/BX C0531A.pdf

15. SPSS Inc. (2007): SPSS for Windows, Version 16.0. Chicago, SPSS Inc. available from:http://www.unimuenster.de/imperia/md/conte nt/ziv/service/software/spss/handbuecher/englisch/ spss_brief_guide_16.0.pdf

16. Suwansaksri J and Wiwanitkit V. (2000): Urine trans, trans-muconic acid determination for monitoring of benzene exposure in mechanics. Southeast Asian J Trop Med Public Health; 31(3): 587-596.

17. Pan C, Chan C, Huang Y, et al. (2008): Urinary 1hydroxypyrene and malondialdehyde in male workers in Chinese restaurants. Occup Environ Med.; 65(11): 732-735.

18. Winn L. (2003): Homologous recombination initiated by benzene metabolites: a potential role of oxidative stress. Toxicological Sciences; 72:143149.

19. Emara A and El-Bahrawy H. (2008): Green Tea Attenuates Benzene-Induced Oxidative Stress in Pump Workers. Journal of Immunotoxicology; 5(1): 69-80.

20. Abou El-Magd S, El-Gohary S, Hammam R, et al. (2010): Biological Assessment of exposure to Benzene among Petrol Stations' Workers in Zagazig City by using Trans,trans-Muconic Acid as Urinary Indicator. Egyptian Journal of Occupational Medicine; 34(2):171-181.

21. Chen Y. (1992): Effects of benzene on lipid peroxidation and the activity of relevant enzymes in humans. Chin J Prevent Med; 26:336-338.

22. Georgieva T, Michailova A, Panev T, et al. (2002): Possibilities to control the health risk of petrochemical workers. Int Arch Occup Environ Health; 75:21-26.

23. Kum C, Sekkin S, Kiral F, et al. (2007): Effects of xylene and formaldehyde inhalations on renal oxidative stress and some serum biochemical parameters in rats. Toxicol Ind Health; 23(2):115120.

24. Karagozler A, Mehmet N and Batcioglu K. (2002): Effects of long term solvent exposure on blood cytokine levels and antioxidant enzyme activities in house painters. Toxicol Environ Health Part A; 65(17):1237-1246.

25. Moro A, Charão M, Brucker N, et al. (2013): Genotoxicity and oxidative stress in gasoline station attendants. Mutat Res.; 754(1-2):63-70. Available online from: http://www.ncbi.nlm.nih.gov/pubmed/23628435

26. Abdallah H, Hussein A, Abdel-Latif, et al. (2010): Ventilatory function and oxidative- antioxidant Status in shoe makers. Science Journal; 2(4):5966. Available online from: http://www.sciencepub.net/researcher/research020 $4 /$ 
27. Rekhadevi P, Rahman M, Mahboob M, et al. (2010): Genotoxicity in Filling Station Attendants Exposed to Petroleum Hydrocarbons. Ann. Occup. Hyg.; 54(8): 944-954.

28. Al-Helaly L. (2011): Some Antioxidant Enzymes in Workers Exposed to Pollutants. Raf. Jou. Sci.; 22(2): 29-38.

29. Kim J, Moon J, Park E, et al. (2011): Changes in Oxidative Stress Biomarker and Gene Expression Levels in Workers Exposed to Volatile Organic Compounds. Industrial Health; 49: 8-14.

30. Nielsen F, Mikkelsen B, Nielsen J, et al. (1997): Plasma malondialdehyde as biomarker for oxidative stress: reference interval and effects of life-style factors. Clinical Chemistry; 43(7): 1209_ 1214.

31. Casado A, Castellanos A, López-Fernández M, et al. (2011): Determination of oxidative and occupational stress in palliative care workers. Clinical Chemistry and Laboratory Medicine; 49(3):471-477.

32. Bolzan A, Bianchi M and Bianchi N. (1997): Superoxide dismutase, catalase and glutathione peroxidase activities in human blood: influence of sex, age and cigarette smoking. Clin Biochem; 30(6):449-454.
33. Yoshie Y and Ohshima H. (1997): Synergistic induction of DNA strands breakage by cigarette tar and nitric oxide. Carcinogenesis; 18: 1359-1422.

34. Jain A, Agrawal B , Varma M, et al. (2009): Antioxidant status and smoking habits: relationship with diet. Singapore Med J; 50(6): 624-631.

35. Burlakova E, Zhizhina G, Gurevich S, et al. (2010): Biomarkers of oxidative stress and smoking in cancer patients. J Can Res Ther [cited 2013 Jul 3]; 6:47-53. Available from: http://www.cancerjournal.net/text.asp?2010/6/1/47 163569

36. Vargas H, Nunes S, de Castro M, et al. (2013): Oxidative stress and inflammatory markers are associated with depression and nicotine dependence. Neurosci Lett; 544:136-40. Available online from: http://www.ncbi.nlm.nih.gov/pubmed/23583694

37. Cernak I, Savic V, Kotur J, et al. (2000): Alterations in magnesium and oxidative status during chronic emotional stress. Magnes Res.;13(1):29-36.

38. Sivonová M, Zitnanová I, Hlincíková L, et al. (2004): Oxidative stress in university students during examinations. Stress ; 7(3):183-191. 


\section{تقييم الاكسدة و حالة مضادات الأكسدة بين عمال محطات الوقود المعرضين للبنزين في مدينة الزقازيق}

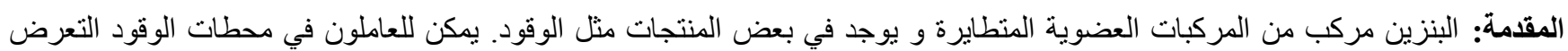

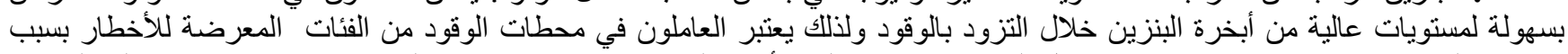

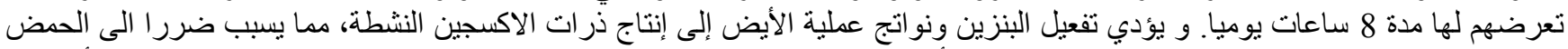

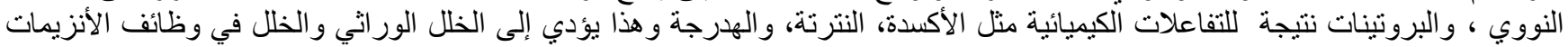

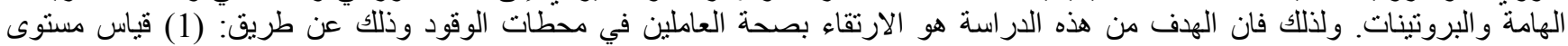
trans,trans-muconic acid Malondialdehyde

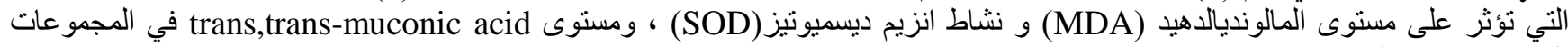

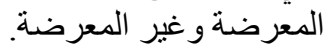
طرق البحث: أجريت هذه الدراسة الميدانية على قطاع مستعرض من العمال في محطات الوقود بمدينة الزقازيق محافظة الثرقية وكذلك عينة

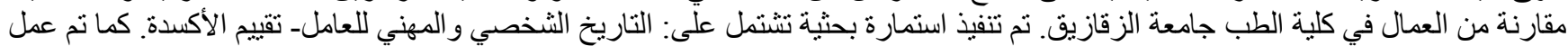

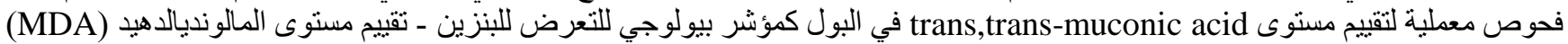

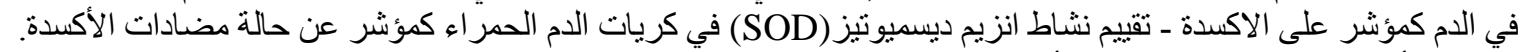

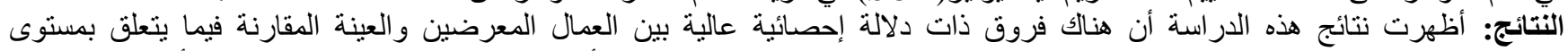

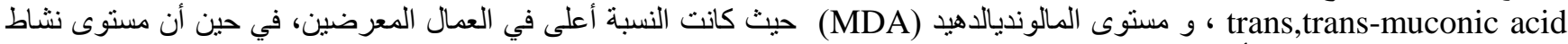
انزيم ديسميوتيز (SOD) كان أقل في العمال المعرضين.

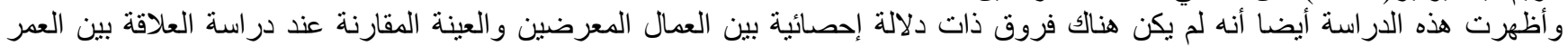

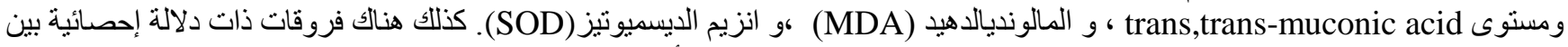

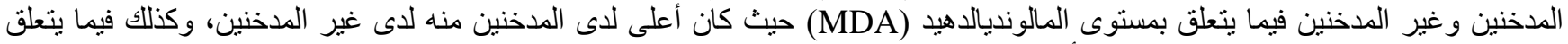

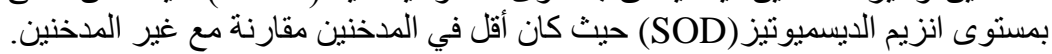

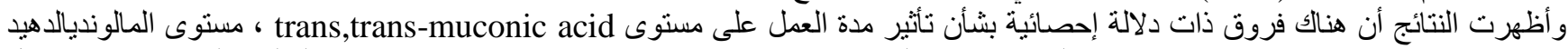

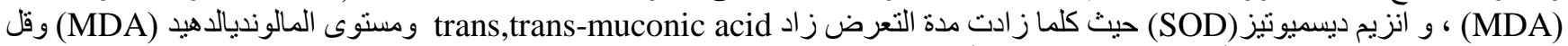

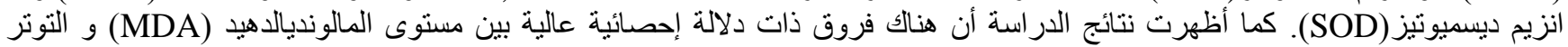

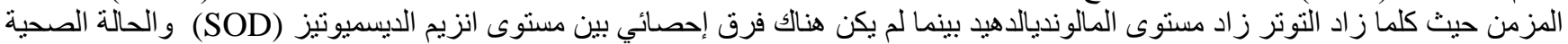

لعمال محطات الوقود.

الخلاصة: كان و اضحا من هذه الدر اسة أن العمل بمحطات الوقود هو مصدر مهم للتعرض لمادة البنزين وهذا كان وان واضحا من حقيقة أن العمال

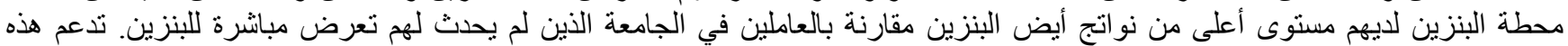

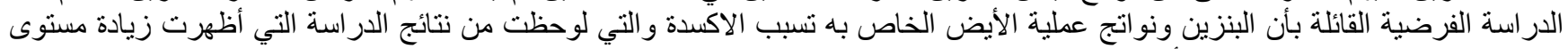

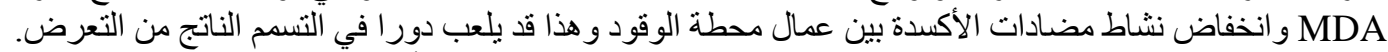

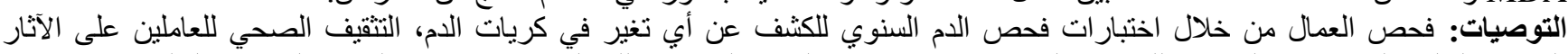

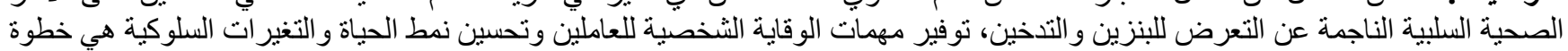
أساسية نحو زيادة المستوى الصحي ونجية التعضب الإجهاد المزين. 